\title{
BioCosmos
}

\section{BIOCOSMOS: NEW PERSPECTIVES ON THE ORIGIN AND EVOLUTION OF LIFE}

\author{
Anthony H. Futerman ${ }^{1, *}$, Siegfried Scherer ${ }^{2}$, Joel L. Sussman ${ }^{3}$, David Snoke ${ }^{4}$, Steve Fuller ${ }^{5}$ \\ ${ }^{1}$ Department of Biomolecular Sciences, Weizmann Institute of Science, Rehovot 7610001, Israel \\ ${ }^{2}$ Core Facility Microbiome, TUM School of Life Sciences, Technical University of Munich, 85354 Freising, Germany \\ ${ }^{3}$ Department of Chemical and Structural Biology, Weizmann Institute of Science, Rehovot 7610001, Israel \\ ${ }^{4}$ Department of Physics and Astronomy University of Pittsburgh, Pittsburgh, PA 15216, USA \\ ${ }^{5}$ Department of Sociology, University of Warwick, Coventry CV4 7AL, United Kingdom
}

As editors, we are delighted to announce the launch of 'BioCosmos: New perspectives on the origin and evolution of life', which, as the name implies, aims to present a wide variety of novel perspectives on the origins and nature of life that go beyond the standard neo-Darwinian paradigm of biological evolution.

Launching a new scientific journal, when the number of journals seems to multiply exponentially every few years, is not a task that the editors undertook lightly. However, all the editors are somewhat frustrated by the lack of genuine discussion in their respective disciplines (which range from biology, to biochemistry, to physics, to philosophy) about theoretical and experimental limitations of current understanding of pathways leading from the origin of life to the evolution of complex life forms. With this in mind, the editors feel that a new journal focusing on the science and the philosophical ramifications of evolution, is warranted. Indeed, classical neo-Darwinian pathways have been challenged by others in recent years, both in scientific conferences [such as the Royal Society meeting in 2016 (https://royalsociety.org/ science-events-and-lectures/2016/11/evolutionary-biology/)] and by the appearance of such organizations as the 'Third Way of Evolution' (https://www.thethirdwayofevolution.com).

Consequently, the editors believe that there are many open and unresolved questions emanating from studies of the origin and evolution of life that have raised critical issues which need to be urgently addressed through open debate.
BioCosmos aims to provide a platform for such discussion. It seeks to publish studies that not only support but also criticize the Darwinian paradigm and current views on the origin of life (for a full list of topics to be covered, please see 'Aims and Scope' at https://sciendo.com/journal/BIOCOSMOS). In the spirit of Newton's 'hypotheses non fingo', BioCosmos will judge the theoretical claims proposed by authors in terms of the evidence placed before the reader, rather than presuming that the authors' ideas and data should fit within a prescribed theoretical framework.

The editors are committed to turning over submissions within as short a time as possible without compromising the quality of peer review. Papers will be handled by one of the editors listed on this editorial, and sent for review by an editorial board member (see full list of editorial board members at https://sciendo.com/journal/BIOCOSMOS) and by at least one additional qualified academic researcher. The range of research potentially covered by BioCosmos is very wide, and the editors are committed to identifying suitable referees to review all submissions as fairly as possible. Manuscripts will also be published as rapidly as possible on the journal website after acceptance.

We trust that you will choose to submit a manuscript to BioCosmos, and we look forward to a genuine exchange of views on new and novel perspectives related to the origin and evolution of life. 